\title{
Ecological Economics: Zur Einführung
}

Die Ecological Economics (ökologische Ökonomie) hat sich ungefähr vor zehn Jahren zu einer eigenständigen Denkrichtung oder Teildisziplin innerhalb der Ökonomie entwickelt. Die VertreterInnen dieser Denkrichtung grenzen sich in ihrem Selbstverständnis von der neoklassischen Umwelt- und Ressourcenökonomie ab, da sie diese in vielerlei Hinsicht als zu reduktionistisch finden. Da inzwischen analytische Erkenntnisse sowie methodische Ansätze vorliegen, deren Ergebnisse auch zunehmend politischen Einfluß erlangen, ist es an der Zeit, den wissenschaftlichen Erkenntnisfortschritt dieses Forschungsgebietes einmal zusammenfassend im Informationsdienst vorzustellen und zu diskutieren: Was verbirgt sich hinter dem Begriff ökologische Ökonomie, der zur Zeit auch in Deutschland in Mode kommt? Was ist der Unterschied zur Umweltund Ressourcenökonomie? Welche neueren Erkenntnisse sind mit diesem Ansatz gewonnen worden?

\section{Rückblick}

Die Herausbildung der ökologischen Ökonomie unterlag wie jede Theorieentwicklung einem Entwicklungsprozeß, bei dem Persönlichkeiten sowie gesellschaftliche Entwicklungen und Wahrnehmungen von Problemen eine Rolle spielen. Namhafte VordenkerInnen des Ansatzes der ökologischen Ökonomie sind Boulding (1966) »The economics of the coming spaceship earth «; Georgescu-Roegen (1971) »The Entropy Law and the Economic Problem«; Daly (1972)»The steady-state Economy «; Meadows, et al. (1972) »Limits to Growth«; Ehrlich, P. und Ehrlich A. (1970) » Population, Ressources, Environment « und Odum (1971) »Environment, Power and Society«. In den 80er Jahren wurden, ausgehend von der Wachstumsdebatte der 70er Jahre und einer zunehmenden Internationalisierung der Umweltprobleme, neue Zielorientierungen sowie neue Ansätze und Methoden zur Problemlösung der Bevölkerungsentwicklung, Umweltproblematik und sozialer und ökonomischer Ungleichheit gesucht. Denn die bisherigen Konzepte sowie Analysemethoden schienen keine hinreichenden Antworten mehr zu liefern. Seit 1982 fanden auf internationaler Ebene verschiedene Workshops und Konferenzen (Stockholm, Barcelona, Ohio) zum Thema »Integration von Ökonomie und Ökologie« statt. Außerdem wurde Anfang der 80er Jahre von der World Commission on Environment and Development die sogenannte Brundtland-Kommission, benannt nach ihrer Vorsitzenden, eingesetzt, die 1987 ihren Bericht vorlegte und den Begriff Sustainable Development einführte. Dieser Begriff ist seit der UNKonferenz 1992 in Rio de Janeiro sehr populär geworden, harrt aber noch der Operationalisierung.

1988 haben die WissenschaftlerInnen, die die Frage nach den Möglichkeiten einer analytischen und methodischen Verzahnung von Ökologie und Ökonomie in den Mittelpunkt ihrer Arbeiten gestellt haben, die International Society for Ecological Economics in den USA gegründet. »Ecological Economics addresses the relationships between ecosystems and economic systems in the broadest sense « (Costanza 1989). Anspruch ist es, die jeweilige Fachdisziplin zu erweitern und »(to) encourage new ways of thinking about the linkages between ecological and economic systems « (Costanza 1989). Ein Forschungsziel für die VertreterInnen der ökologischen Ökonomie besteht in der Entwicklung von Methoden und Strategien für die Operationalisierung von $\mathrm{Su}$ stainable Development.

\section{Voranalytische Visionen}

Abgrenzen läßt sich die ökologische Ökonomie gegenüber der Umwelt- und Ressourcenökonomie zum einen durch die voranalytischen Visionen und zum anderen durch eine methodische und normative Erweiterung der jeweiligen Fachdisziplin.

\section{Scale}

Forschungsgegenstand der ökologischen Ökonomie ist das Verhältnis zwischen dem »Ökosystem « und dem ökonomischen System im weitesten Sinne. Es wird davon ausgegangen, daß die Wirtschaft in ihrer biophysikalischen Dimension ein offenes Subsystem eines endlichen, nicht wachsenden und materiell geschlossenen $\mathrm{Ge}$ samtsystems »Erde« ist. »Offen« deshalb, weil das Wirtschaftssystem Materie und Energie von der natürlichen Umwelt in niedrig-entropischer Form aufnimmt und in hoch-entropischer Form wieder an die Umwelt abgibt. Dieser Verarbeitungsprozeß wird als (Stoff-) Durchsatz (throughput) bezeichnet. Der Durchsatz ergibt sich aus der Bevölkerungszahl mal dem Verbrauch an Energie und Materie. Demnach ist eine Wirtschaft naturverträglich, wenn der Durchsatz konstant und im Einklang mit dem umgebenen System ist, d.h. die Regulierungsfähigkeit und Assimilationsfähigkeit der Natur werden berücksichtigt (Daly 1994). Damit wird quantitatives Wirtschaftswachstum in Frage gestellt.

\section{Interdependenz}

Ferner wird davon ausgegangen, daß die Natur für den Menschen mehrere Funktionen erfüllt. Zum einen stellt sie Bestände regenerierbarer und nichtregenerierbarer Ressourcen zur Verfügung. Sie sind die Grundlage für natürliche Dienstleistungen (Life-Support-Function) der Natur. Zum anderen hat die Natur eine ästhetische Bedeutung für den Menschen. Diese Funktionen sind rekursiv miteinander gekoppelt sowie mit dem Subsystem »Wirtschaft«. Sie kennzeichnen die Interdependenz des ökologischen und ökonomischen Systems und ihre jeweilige Dynamik. Ökologische Grenzen, die aus der Notwendigkeit der Erhaltung dieser Funktionen resultieren, werden von der ökologischen Ökonomie anerkannt. Aufgrund dieser Sichtweise wird ein Komplementärverhältnis zwischen der Natur oder natürlichem Kapital und dem menschengemachten Kapital unterstellt. Die neoklassische Substitutionsthese zwischen »natürlichem « Kapital und menschengemachten Kapital wird nur eingeschränkt akzeptiert.

\section{Zeit}

Aufgrund der Systemkomplexität und Systemdynamik der Natur wird dem Markt- und Preismechanismus nicht zugetraut, die ökologischen Grenzen zu erfassen und entsprechende Knappheitssignale zu setzen. Denn viele Umweltnutzungen werden zeitverzögert wahrgenommen aufgrund von Akkumulationsprozessen, rekursiven Prozessen und räumlicher Diffusion. Außerdem können Umweltnutzungen zu irreversiblen Schäden führen. Umgekehrt reagiert das ökonomische System innerhalb schnellerer Anpassungszeiten und mit kürzeren Zeithorizonten. Deshalb ist in Modellen »Zeit« als. Variable explizit zu berücksichtigen.

\section{Unsicherheit}

Die Systemkomplexität der Ökologie sowie die Unwissenheit darüber führen zu Entscheidungen unter Unsicherheit. Deshalb wird von einer prozeßhaften Rationalität, die Lernprozesse einschließt, ausgegangen. Referenzmaßstab ist in erster Linie eine kollekțive Rationalität. Das Individuum steht nicht im Mittelpunkt der Analyse, sondern die Gesellschaft (generationsübergreifend) als ganzes. Ferner gilt ein technologisch pessimistisches Vorsichtsprinzip: technologischer Fortschritt wird nicht als alleinige Lösung des Umweltproblems gesehen, da einige Probleme als nicht durch den Einsatz von Technik lösbar angesehen werden bzw. ihr Einsatz wiederum neue und andere Risiken mit sich bringen kann kann (Atom, FCKW, Gentechnik).

\section{Gerechtigkeit}

Wenn ökologische Grenzen akzeptiert und gesetzt werden, dann werden Verteilungsprobleme zentral, die bislang in der Umwelt- und Ressourcenökonomie als nachrangig im Vergleich zum Effizienzproblem gesehen wurden. Hierbei sind intra- und intergenerative Verteilungsprobleme nicht voneinander zu trennen. Im Rahmen der ökologischen Ökonomie werden Werturteile der Gerechtigkeit thematisiert, wobei auch vor einer Diskussion der Umverteilung aus Effizienzgründen (Daly 1994) nicht zurückgeschreckt wird. Denn nach den heutigen Erkenntnissen kann Wohlstand und Wohlfahrt nicht mehr durch quantitatives Wirtschaftswachstum global erreicht werden. Dabei wird das Ressourcen- und Umweltproblem nicht ausschließlich als ein Allokationsproblem aufgefaßt, sondern nach Daly (1992) auch als Distributions- und Scale-Problem. 


\section{Methodik}

\section{Scale}

Die Ansätze, die sich mit dem ökonomischen und ökologischen Niveau oder Scale beschäftigen, haben einen ökologischen, naturwissenschaftlichen Schwerpunkt. Analyseschwerpunkt ist eine Verknüpfung des ökonomischen und ökologischen Systems mit Hilfe von Input/Output-Beziehungen. Es wird aber kein Gleichgewicht im ökonomischen Sinne angestrebt, sondern ein evolutorischer Prozeß der Stabilisierung in beiden Systemen. Es lassen sich zwei Modellierungsansätze unterscheiden, die bislang eher einen heuristischen Charakter haben. Die einen beschäftigen sich mit der Struktur der ökonomischökologischen Interdependenz (Ansätze der Thermodynamik). Ausgangspunkt ist die Arbeit von Georgescu-Roegen (1971), der die Hauptsätze der Thermodynamik (Physik), insbesondere den zweiten Hauptsatz, auf die physikalischen Grundlagen der Ökonomie anwendete. Als thermodynamische Modelle werden modellmäßige Darstellungen bezeichnet, die thermodynamische Größen wie Energie, Entropie, Stoffmengen sowie ihre Flüsse als wesentliche Elemente enthalten. Diese Ansätze stellt Georg Meran in seinem Beitrag vor.

Die anderen Modellansätze untersuchen die Evolution der ökologisch-ökonomischen Interaktion (Populationsdynamik biologischer Systeme). Die Bio-Economics betrachtet die dynamische Entwicklung von interagierenden Systemen. Ansatzpunkt sind die auf Lotka und Volterra zurückgehenden Räuber-Beute-Modelle aus der Biologie, die vorwiegend in der Ressourcenökonomie angewendet werden. Mit diesen Modellen können Drohpunkte als Niveaueffekte (Bevölkerung und Ressourcenverbrauch) für die einzelnen Systeme bestimmt werden. Allerdings werden keine feedback-Effekte zwischen dem ökonomischen und ökologischen System berücksichtigt. Weitere Modellierungsansätze sind Überlegungen der evolutorischen Ökonomik. Sie greift auf evolutionstheoretische, chaostheoretische und synergetische Ansätze zurück. Analyseschwerpunkt ist die Selbstorganisation von ökonomischen und ökologischen Systemen. Den Ansatz der evolutorischen Ökonomik stellt Markus Parsche vor.

Die vorgestellten Modellansätze verwenden die Mathematik nicht-linearer Systeme und basieren vielfach auf Computersimulationsmodellen. Ergebnisse dieser Modelle sind (Perrings 1995):

- Das ökonomische und ökologische System sind interdependent. Das Niveau (Scale) des ökonomischen Systems hat einen erheblichen Einfluß auf das ökologische System.

- Die Dynamik beider Systeme ist durch Diskontinuitäten und Drohpunkte geprägt.

- Die Stabilität beider Systeme hängt von ihrer jeweiligen Selbstorganisationsfähigkeit bei Stre $\beta$ und Schocks ab.

\section{Allokation}

Pragmatische Arbeiten untersuchen, wie die oben genannten Ergebnisse die Bewertung von natür-

\section{The International Society for Ecological Economics (ISEE)}

Die ISEE wurde 1988 gegründet, um die Integration von Ökologie (nature's household) und Ökonomie (humanity's household) zu fördern. Zu diesem Zweck gibt sie die Zeitschrift »Ecological Economics« (12 mal im Jahr) und für die Mitglieder »The Ecological Economics Bulletin « (4 mal im Jahr) heraus. Ferner veranstaltet sie regelmäßig wissenschaftliche Tagungen und Workshops.

Wichtige Veröffentlichungen sind

- Ecological Economics: The Science and Management of Sustainability. Robert Costanza (Ed. ), Columbia University Press 1991

- Investing in Natrual Capital: The Ecological Economics Approach to Sustainability. AnnMari Jannsson et al. (Eds. ), Island Press 1994

lichen Ressourcen für eine effiziente Nutzung beeinflussen. Für die Nutzungs- und Bewertungsfragen wird vielfach die Methodik der Umwelt- und Ressourcenökonomik verwendet, allerdings auf neue Anwendungsfelder ausgedehnt. So steht die Bewertung von ökologischen Ressourcen, von einzelnen Tier- und Pflanzenarten, Ökosystemen und globalen Problemen im Mittelpunkt der Analyse. Ermittelt werden die Opportunitätskosten der Umweltdegradierung für heutige und zukünftige Generationen. Allerdings werden hier ökologische Grenzen wie der Safe Minimum Standard oder eine Carring Capacity akzeptiert und gesetzt. Unter den Bewertungsmethoden nimmt die Analyse der Zahlungsbereitschaft einen zentralen Platz ein (Hampicke 1995).

\section{Distribution}

Nach modelltheoretischen Erkenntnissen ( $\mathrm{Ho}^{-}$ warth und Norgaard 1991) wird deutlich, daß Effizienz kein hinreichendes Kriterium für eine intergenerative Gerechtigkeit bei dem Ziel einer nachhaltigen Entwicklung ist. Deshalb wird versucht, in die Modelle zu Allokations- und Verteilungsfragen das Rawls-Kriterium »Schleier des Unwissen « (Minmax), den kategorischen Imperativ »Handele so wie Du erwartest, daß andere Handeln sollen « oder das Prinzip der Verantwortung nach Jonas »Verantwortung gegenüber der Natur und zukünftigen Generationen «, zu integrieren. Auf Probleme der intergenerativen $\mathrm{Ge}-$ rechtigkeit geht Hans G. Nutzinger in diesem Schwerpunkt ein.

\section{Ausblick}

Mit Hilfe der ökologischen Ökonomie sind Erkenntnisse gewonnen worden, zu denen die Umwelt- und Ressourcenökonomie nicht in der Lage war. Denn letztere hat das Umwelt- sowie das Ressourcenproblem jeweils als Einzelproblem begriffen und keine Verknüpfungen hergestellt. Zwar versucht die »Mainstream-Ökonomie «nun, die Erkenntnisse der ökologischen Ökonomie in
- Toward Sustainability. Concepts, Methods, \& Policy. Jeroen C. J. M. van den Bergh, Jan van der Straaten (Eds.), Island Press 1994

- Natural Capital and Human Economic Survival. Thomas Prugh (Ed.), im Erscheinen

- Getting Down to Earth. Practical Applications of Ecological Economics. Olman Segura, Juan Martinez-Alier et al. (Eds.), im Erscheinen

The International Society for Ecological Economics, P. O. Box 1589, Solomons, Maryland 20688 USA, Tel. : (410) 326-0794, Fax (410) 326-7354 - Wer sich über den aktuellen Stand der Diskussion innerhallb der Ecological-Economics-Gemeinde informieren oder selbst aktiv daran teilnehmen will, kann dies über das Internet tun (http: / / csf. colorado. edu / ecolecon /index. html).

ihre Modelle zu integrieren, aber hervorgebracht hat sie sie nicht.

Die ökologische Ökonomie hat sich bislang sehr stark auf die Scale-Problematik konzentriert. Wenig Beachtung haben dabei Fragen der Verarbeitung ökologischer Grenzen in gesellschaftlichen Systemen gefunden. Es gibt zwar eine breite Diskussion zur Bewertungsproblematik, aber Fragen der Nutzungsrechte (property rights), von Indikatoren für Nachhaltigkeit und nach geeigneten Institutionen (was sind nach dem Leitbild Sustainable Development geeignete Unterziele oder was sind geeignete Strategien für einen Strukturwandel), haben bislang weniger Beachtung gefunden. Mit anderen Worten: ökologische, soziale und ökonomische Zielkonflikte gilt es zu benennen und Strategien zur Auflösung dieser Konflikte zu entwickeln. Einige dieser Zielkonflikte werden in den Aufsätzen von Paul Klemmer, Frank Beckenbach und Mathias Binswanger thematisiert. Diese Zielkonflikte werden Gegenstand zukünftiger Forschungsfragen sein, um Strategien und politische Handlungsempfehlungen zur ihrer Auflösung zu entwickeln und um so einer nachhaltigen, zukunftsfähigen Entwicklung näherzukommen.

\section{Literatur}

Birgit Soete, Berlin

- Costanza, R. (1989): What is Ecological Economics?, in: Ecological Economics, vol. 1, No 1, S. 1 $-8$

- Daly, H. (1992): Allokation, distribution, and scale: towards an economics that is efficient, just and sustainable, in: Ecological Economics, S. 185-193

- Daly, H. (1994): Ökologische Ökonomie: Konzepte, Fragen, Folgerungen. In: Jahrbuch Ökologie 1995 , S. $147-161$

- Hampicke, U. (1995): Ökologische Ökonomie. In: Junkernheinrich, M. et al.: Handbuch zur Umweltökonomie, S. 138 - 144

- R. Howarth, R. Norgaard (1991): Sustainability and Discounting the Future. In: R. Costanza: Ecological Economics. S. 88-1011

- Perrings, C. (1995): Ecology, Economics and Ecological Economics, in: Ambio, vol. 24, No 1, S. 60 64 
(c) 20I0 Authors; licensee IÖW and oekom verlag. This is an article distributed under the terms of the Creative Commons Attribution Non-Commercial No Derivates License (http://creativecommons.org/licenses/by-nc-nd/3.o/), which permits unrestricted use, distribution, and reproduction in any medium, provided the original work is properly cited. 